\title{
Serotonin Syndrome: Fairly Common but Frequently Forgotten
}

\author{
Awad Magbri, MD, FACP*, Mariam El-Magbri, BSC, MPH, Kamran Suljuki, Phama D, MBA, and Darcy \\ Rose, $R N$
}

Toledo Vascular Access Center, Toledo Clinic Inc, USA

\begin{abstract}
The authors presented a case of serotonin syndrome cause by drug interaction. The patient is presented with classical features of the syndrome with hyper-excitability, tremor, change in mental status, and low grade fever. The patient made uneventful recovery after the stoppage of the offending drugs. This case calls for the clinicians to be vigilant of the drug interaction that can cause unintended side effects to the patient under their care.
\end{abstract}

\section{Keywords}

Serotonin syndrome, Hyper-excitability, Autonomic hyperactivity, Tremor

\section{Case History}

A 72-year-old Caucasian man has past medical history of hypertension, type- 2 diabetes mellitus, peripheral vascular disease, hyperlipidemia, and anxiety-depression mood. He was admitted to the hospital secondary to fall which resulted into left neck of femur fracture. He underwent successful open reduction and internal fixation. He was on escitalopram and bupropion hydrochloride before the surgery for his anxiety/depression. He was treated with tramadol by the orthopedic surgeon for his postoperative pain. The patient was transferred to long-term acute care facility (LTAC) for continuation of his medical management and rehabilitation. Postoperatively, about three days later he started having agitation, tremor of his hands, and hyper-excitability with hyperreflexia, confusion and low grade fever. These symptoms were attributed to serotonin syndrome, and they resolved only when escitalopram, bupropion, and tramadol were discontinued.

In the LTAC his vital signs were, blood pressure 161/85 $\mathrm{mmHg}$, heart rate of $103 / \mathrm{min}$ regular, respiratory rate 20 / min, temperature of $99.8 \mathrm{~F}$, and oxygen saturation was $98 \%$ on $3 \mathrm{~L}$ nasal cannula. He demonstrated clear encephalopathic picture with agitation, tremor, confusion, and hyper-reflexia with ankle clonus bilaterally. His pupils were dilated with sluggish reaction to light, but ocular clonus could not be elicited. His respiratory system examination was unremarkable. His abdominal examination reveals no tenderness, masses or hepato-splenomegaly. However, his bowel sounds were hyperactive. His surgical wound was clean with no signs of infection and his lower limb examination showed only stasis dermatitis with no tenderness or edema. Magnetic resonance imaging of the brain for perfusion (MRI-perfusion) study was not performed. Upon admission to the hospital his laboratory data showed sodium of $142 \mathrm{mE} / \mathrm{L}$, potassium of $4.8 \mathrm{mEq} / \mathrm{L}$, chloride of $100 \mathrm{mEq} / \mathrm{L}$, bicarbonate $27 \mathrm{Mmol} / \mathrm{L}$, blood urea nitrogen of $29 \mathrm{mg} / \mathrm{L}$. and serum creatinine of $0.92 \mathrm{mg} / \mathrm{L}$. He was hypoglycemic with a blood sugar level of $63 \mathrm{mg} / \mathrm{L}$ with no change in his mental status after correction of his hypoglycemia. His calcium rate is of $8.9 \mathrm{mg} / \mathrm{L}$ and complete blood count showed white cell count of 7.01 with hemoglobin of 8.2 and hematocrit of 27.9, and his platelet count was $211 \mathrm{~K} / \mathrm{L}$. His blood culture and urine culture were negative.

There was no clear explanation of his acute confusional state with hyper-excitability and low grade fever which persisted even after treatment of hypoglycemia. After reviewing his medication the only new addition to his medication was tramadol. Escitalopram is a selective serotonin reuptake inhibitor and the opioid tramadol also has serotonergic effects, so the combination could result in the SS. The possibility of drug interaction with serotonin toxicity was raised and escitalopram, bupropion hydrochloride, and tramadol were stopped. The hospital course of the patient after discontinuation of his medication shows rapid improvement and returned to baseline in his physical and mental health. His confusional state had cleared slowly giving credence to the clinical diagnosis of serotonin toxicity.

*Corresponding author: Awad Magbri, MD, Toledo Vascular Access Center, Toledo Clinic Inc, Toledo, OH, USA, Tel: 815-5208211

Accepted: March 09, 2019

Published online: March 11, 2019

Citation: Magbri A, El-Magbri M, Suljuki K, et al. (2019) Serotonin Syndrome: Fairly Common but Frequently Forgotten. Ann Nephrol 4(1):33-35 


\section{Case Discussion}

Serotonin toxicity or serotonin syndrome (SS) is a lifethreatening condition associated with increased serotonergic activity in the central and peripheral nervous systems [1]. The syndrome is usually results from misuse of therapeutic medications, inadvertent drug interaction, or intentional selfpoisoning [1].

Serotonin syndrome is characterized by a triad of mental status changes, neuromuscular abnormalities, and autonomic hyperactivity [1-4]. SS is a spectrum of clinical activity ranges from benign clinical findings to lethal condition [1-4]. The syndrome occurs in all age groups and the incidence of this syndrome increase with the upsurge use of serotonergic drugs $[1,4,5]$. The syndrome can be ascribed to overdose $[6,7]$ and can even occurs in patients who are treated with therapeutic hypothermia after cardiac arrest [8].

The selective serotonin reuptake inhibitors (SSRI) are the most implicated drugs in SS [9]. Serotonin modulates attention, behavior, and thermoregulation in the central nervous system (CNS). In the peripheral nervous system, serotonin is produced by intestinal enterochromaffin cells and is involved in the regulation of vasoconstriction, uterine contraction, bronchospasm, and gastrointestinal motility [3]. Serotonin is also found in platelets and promotes platelet aggregation.

Serotonin syndrome can result from the following mechanisms of drugs:

1. Increases serotonin formation e.g. tryptophan.

2. Increase release of serotonin- amphetamines, cocaine, MDMA (ecstasy), levodopa, carbidopa-levodopa.

3. Impair reuptake from the synaptic cleft into the pre-synaptic neuron - cocaine, MDMA, meperidine, tramadol, pentazocine, SSRIs, serotonin-norepinephrine reuptake inhibitors (SNRIs, e.g. duloxetine which can cause SS even after single dose [10], dopamine-norepinephrine reuptake inhibitors (bupropion), serotonin modulators (trazodone, vilazodone), tricyclic antidepressants (amitriptyline, clomipramine, desipramine, nortriptyline, protriptyline), 5-HT3 receptor antagonists (ondansetron), metoclopramide, valproate, carbamazepine, dextromethorphan.

4. Inhibits serotonin metabolism (i.e., inhibits monoamine oxidase activity) - MAOIs (phenelzine, selegiline, linezolid, procarbazine).

5. Direct serotonin agonist - buspirone, triptans (sumatriptan, rizatriptan), ergot derivatives (ergotamine, methylergonovine), fentanyl, LSD.

6. Increase sensitivity of postsynaptic receptors - lithium.

Stimulation of the postsynaptic 5-HT1A, and 5-HT2A receptors have been implicated in the SS [11], but any drug interaction results in the increase serotonin activity from the above mentioned mechanisms can results to SS. The interactions of MAOI with other drugs may be the most severe and often leads to adverse outcome including death in SS $[12,13]$.
The diagnosis of SS is mainly based on clinical grounds which involves in increase of serotonergic activity, mental status changes, anxiety, agitation, delirium, restlessness, and disorientation [14]. Autonomic manifestation of diaphoresis, tachycardia, hyperthermia, hypertension, vomiting and diarrhea [3] can be more pronounced in this syndrome. Neuromuscular hyperactivity with tremor, muscle rigidity, myoclonus, and hyperreflexia with positive Babinski sign are particularly common. Lower limbs hyperreflexia, ocular and lower limbs clonus (Video 1 ) and rigidity are often more noticeable [3]. The majority of SS are presented within 24-hours of change in the dose of serotonergic drug or drugdrug interaction [3].

Laboratory evaluation- may reveal non-specific findings like elevated white blood cells, elevated creatinine phosphokinase, decrease serum bicarbonate, disseminated intra-vascular coagulation, rhabdomyolysis, metabolic acidosis, renal failure, myoglobinuria, and acute respiratory distress syndrome [3].

A Diagnostic criteria is based on clinical depiction of the serotonin toxicity which can be enhanced by the use of Hunter Toxicity Criteria Decision Rules [15]. These criteria have high sensitivity and specificity ( $84 \%, 97 \%$ respectively) in a patient who has taken serotonergic agent.

1. Spontaneous clonus

2. Inducible clonus + agitation or diaphoresis

3. Ocular clonus + agitation or diaphoresis

4. Tremor + hyperreflexia

5. Hypertonia + temperature $>38 \mathrm{C}+$ ocular clonus or inducible clonus

The differential diagnosis of SS includes:

1. Neuroleptic malignant syndrome (NMS)

2. Anticholinergic toxicity

3. Malignant hyperthermia

4. Sympathomimetic agent intoxication

5. Sedative-hypnotic withdrawal

6. Meningitis

7. Encephalitis

The treatment of SS consists of:

1. Discontinue serotonergic agents

2. Sedate with benzodiazepines (lorazepam 1-2 mg IV, titrate dose to effects)

3. Provide oxygen to (maintain $\mathrm{SPO}_{2}>94$ ), IV fluids and continuous cardiac monitor

4. If benzodiazepines and supportive care fail to improve agitation and abnormal vital signs, give cyproheptadine (12 mg PO or via NGT)

5. Patients with temperature $>41.1 \mathrm{C}$ with immediate sedation, paralysis, and intubation. 
Back to our patient, following discontinuation of the escitalopram, bupropion hydrochloride, and tramadol, he continued to show fast clinical improvement with resolution of his symptoms within 24 hours. He continued to have physical therapy and occupational therapy with improvement in his strength and was able to put weight on the left leg. In summary, SS should be considered whenever the clinical picture suggested the possibility of SS. Missing the diagnosis of SS can have dire consequence to the patient afflicted by the disease.

\section{References}

1. Boyer EW, Shannon M (2005) The serotonin syndrome. N Engl J Med 352: 1112-1120.

2. Bodner RA, Lynch T, Lewis L, et al. (1995) Serotonin syndrome. Neurology 45: 219-223.

3. Mason PJ, Morris VA, Balcezak TJ (2000) Serotonin syndrome. Presentation of 2 cases and review of the literature. Medicine (Baltimore) 79: 201-209.

4. Birmes P, Coppin D, Schmitt L, et al. (2003) Serotonin syndrome: A brief review. CMAJ 168: 1439-1442.

5. Sternbach H (1991) The serotonin syndrome. Am J Psychiatry 148: 705-713.

6. Canan F, Korkmaz U, Kocer E, et al. (2008) Serotonin syndrome with paroxetine overdose: A case report. Prim Care Companion J Clin Psychiatry 10: 165-167.
7. De Roos FJ (2005) Drug interactions: combinations that can kill your patients. American College of Emergency Physicians Scientific Assembly lecture, September 26, 2005, Washington Convention Center.

8. Fugate JE, White RD, Rabinstein AA (2014) Serotonin syndrome after therapeutic hypothermia for cardiac arrest: A case series. Resuscitation 85: 774-777.

9. Bronstein AC, Spyker DA, Cantilena LR Jr, et al. (2012) 2011 Annual report of the American Association of Poison Control Centers National Poison Data System (NPDS): $29^{\text {th }}$ Annual Report. Clin Toxicol (Phila) 50: 911-1164.

10. Gelener P, Gorgulu U, Kutulu G, et al. (2011) Serotonin syndrome due to duloxetine. Clin Neuropharmacol 34: 127-128.

11. Mills KC (1997) Serotonin syndrome. A clinical update. Crit Care Clin 13: 763-783.

12. Isbister GK, Buckley NA (2005) The pathophysiology of serotonin toxicity in animals and humans: implications for diagnosis and treatment. Clin Neuropharmacol 28: 205-214.

13. Ramsay RR, Dunford C, Gillman PK (2007) Methylene blue and serotonin toxicity: inhibition of monoamine oxidase A (MAO A) confirms a therapeutic prediction. Br J Pharmacol 152: 946-951.

14. Ganetsky M, Brush E (2005) Serotonin syndrome- what have we learned. Clin Ped Emerg Med 6: 103-108.

15. Dunkley EJ, Isbister GK, Sibbritt D, et al. (2003) The hunter serotonin toxicity criteria: Simple and accurate diagnostic decision rules for serotonin toxicity. QJM 96: 635-642.

DOI: $10.36959 / 832 / 396$

Copyright: (C) 2019 Magbri A, et al. This is an open-access article distributed under the terms of the Creative Commons Attribution License, which permits unrestricted use, distribution, and reproduction in any medium, provided the original author and source are credited. 